\title{
On the formation of tritum from deuterium in titanium powder
}

\author{
D.D. Afonichev ${ }^{\dagger}$, T.I. Nazarova \\ †afon@imsp.ru \\ Institute for Metals Superplasticity Problems RAS, 39 Khalturin St., Ufa, 450001, Russia
}

The main proof of fusion reactions of deuterium nuclei is the presence of nuclear reaction products. When detecting tritium after saturation of titanium powders with deuterium reproducible results of its high concentration have been found. Titanium powders of two different batches have been used. According to metallographic studies, powder \# 1 had an average particle size of $d=110 \pm 5 \mu \mathrm{m}$; lazer diffraction analyzer of particle sizes registered one more maximum near the size of $d=0.2 \mu \mathrm{m}$. Powder \# 2 was a sifted fraction with sizes in interval $80<d<150 \mu \mathrm{m}$. For powder \# 1 the measured concentrations of tritum amounted about $c_{\mathrm{T}}=(11-14) \cdot 10^{3} \mathrm{dpm}$ and for powder $\# 2 c_{\mathrm{T}}=(1.45-1.57) \cdot 10^{3} \mathrm{dpm}$, a concentration typical for deuterium used. An effect of the dispersity of initial titanium powder on the proceeding of nuclear reactions has been demonstrated. Earlier, a mechanism of interaction of deuterons in a metallic matrix by means of a resonance transfer of a neutron from one deuteron to the other has been proposed. One of the products of such an interaction is a tritium nucleus. Such a process can run in elongated defects. The cross dimension of such defects was not known, however. From an analysis of measurements of titanium powder particle sizes one can conclude that the optimum size of pores must be about $d_{1}=3 \cdot 10^{-8} \mathrm{~m}$. During all experiments the levels of neutrons $\left(N_{n}=30 \pm 21 / 50 \mathrm{sec}\right)$ and gamma quanta $\left(N_{y}=700 \pm 151 / 5 \mathrm{sec}\right)$ did not exceed the values typical for the room where experiments were carried out.

Keywords: deuterium, tritum, cold fusion.

\section{Об образовании трития из дейтерия в порошке титана}

\author{
Афоничев Д.Д. ${ }^{\dagger}$, Назарова Т.И. \\ †afon@imsp.ru \\ Институт проблем сверхпластичности металлов РАН, ул. Халтурина 39, 450001, Уфа, Россия
}

Основным доказательством реакций слияния ядер дейтерия является наличие продуктов ядерных реакций. При измерении трития после насыщения дейтерием титанового порошка были зарегистрированы воспроизводимые результаты его высокой концентрации. Были использованы порошки титана двух разных партий. Порошок № $1-$ средний размер частиц составлял $d=110 \pm 5$ мкм по металлографическим измерениям, кроме того на лазерном дифракционном анализаторе размеров частиц зарегистрирован еще один максимум с размером $d=0,2$ мкм, и порошок № $2-$ просеянная фракция $(80<d<150$ мкм). Для порошка № 1 измеренные значения концентрации трития составляли $c_{\mathrm{T}}=(11-14) \cdot 10^{3} \mathrm{dpm}$, а для порошка № $2 c_{\mathrm{T}}=(1,45-1,57) \cdot 10^{3} \mathrm{dpm}$, концентрация, обычная для использованного дейтерия. Показано влияние дисперсности исходного титанового порошка на протекание ядерной реакции. Ранее был предложен механизм взаимодействия дейтронов в металлической матрице за счет резонансного переноса нейтрона от одного дейтрона к другому. Одним из продуктов такого взаимодействия будет ядро трития. Такой процесс может протекать в длинномерных дефектах. Остается вопрос о поперечном размере таких дефектов. Из анализа результатов измерения размеров частиц порошка титана можно сделать вывод, что оптимальный размер пор должен составлять $d_{1}=3 \cdot 10^{-8}$ м. Во время всех экспериментов уровни нейтронов $\left(N_{\text {пn }}=30 \pm 21 / 50\right.$ сек $)$ и гамма квантов $\left(N_{\text {пү }}=700 \pm 151 / 5\right.$ сек $)$ не превысили обычные величины для используемого помещения.

Ключевые слова: дейтерий, тритий, холодный ядерный синтез. 


\section{1. Введение}

В текущем году исполнилось 27 лет со дня пресс-конференции, на которой М. Флейшман и С. Понс объявили об открытии ими процесса слияния ядер дейтерия при комнатной температуре [1]. К сожалению, часть положений этой работы оказалась неверной, что дало возможность оппонентам отнести эти исследования к области патологической науки [2]. Тем не менее, изучение низкоэнергетических ядерных реакций в настоящее время продолжается во многих лабораториях США, Японии, Израиля, Китая и т. д. [3].

В этой области исследований существуют две основные проблемы. Во-первых - отсутствие общепризнанного механизма взаимодействия ядер дейтерия в металлической матрице, а во-вторых - плохая воспроизводимость получаемых результатов.

\section{2. Материал и методика эксперимента}

В качестве материала исследования использовали порошок титана марки ПТЭМ-1 (Fe 0,06, C 0,007, Si 0,03, $\mathrm{Cl}$ 0,05, N 0,03) двух разных партий.

Использовалась вакуумная установка на базе турбомолекулярного насоса TURBO-V70 фирмы Varian (США), с помощью которого достигалось остаточное давление в системе до $P=1 \times 10^{-6}$ торр.

В баллон установки объемом $V=2,5$ л. напускали дейтерий, очищенный пропусканием через палладиевый фильтр, до давления $P=0,7$ атм. Во время экспериментов постоянно контролировали уровень нейтронов и гамма квантов с использованием радиометра спектрометрического СРПС-2. Порошок титана помещали в кварцевую трубку и отжигали при температуре $T=600^{\circ} \mathrm{C}$ до остаточного давления в системе $P=3 \times 10^{-5}$ торр. Затем при этой температуре дейтерий из баллона напускали в кварцевую трубку. После достижения давления газа в системе до $P=-0,1$ атм., печь с кварцевой трубки снимали, и далее процесс насыщения протекал при охлаждении порошка на воздухе. Весь процесс поглощения дейтерия до давления $P<1$ торр. занимал не более 15 минут.

По окончании насыщения порошка титана дейтерием порошок извлекали из кварцевой трубки и после тщательного перемешивания часть его $(m=60$ мг) сжигали в токе кислорода с пропусканием продуктов сгорания через жидкий сцинтиллятор. Концентрацию трития измеряли на спектрометрическом радиометре Hidex 300 SL (Финляндия).

Размер частиц порошка титана измеряли на снимках, сделанных на сканирующем электронном микроскопе Tescan Vega 3SBH (Czech Republic) с приставкой энергодисперсионного анализа - X-act (Oxford Instruments) и на лазерном дифракционном анализаторе размеров частиц SALD 7101 фирмы Shimadzu (Япония).

\section{3. Результаты и обсуждение}

Из исследований по термоядерному синтезу известно, что при слиянии ядер дейтерия возможно несколько путей протекания реакции:

$$
\mathrm{D}+\mathrm{D} \quad \begin{array}{lcl}
\rightarrow & { }^{1} \mathrm{n}+{ }^{3} \mathrm{He} & 3,3 \text { Мэв } \\
\rightarrow & { }^{1} \mathrm{p}+{ }^{3} \mathrm{~T} & 4,0 \text { Мэв } \\
\rightarrow & { }^{4} \mathrm{He}+\gamma & 23,8 \text { Мэв }
\end{array}
$$

здесь ${ }^{1} \mathrm{n}$ и ${ }^{1} \mathrm{p}-$ это нейтрон и протон соответственно, ${ }^{3} \mathrm{~T}$ - тритий, ${ }^{3} \mathrm{He}$ и ${ }^{4} \mathrm{He}$-изотопы гелия, $\gamma$ - гамма квант, Мэв - единица измерения энергии в ядерной физике (1 Мэв = 1,6 $\times 10^{-13}$ дж).

Попытки зарегистрировать нейтроны разными группами исследователей по всему миру, а также нашими экспериментами, выявили отсутствие нейтронных потоков высокой плотности [3,4]. А это означает, что - либо явление отсутствует, либо механизм реакции слияния ядер дейтерия отличается от протекания известного для термоядерного синтеза.

Ранее был предложен механизм [5], по которому возможно образование трития из дейтерия по реакции (2). Предполагается, что реакция происходит на длинномерных дефектах кристаллического строения за счет переноса нейтрона от одного дейтрона к другому с образованием тритона и протона.

Основным аргументом оппонентов «холодного ядерного синтеза» является отсутствие продуктов ядерных реакций, поэтому основной задачей наших исследований последних лет была наработка высокой концентрации трития.

Во время проведения экспериментов, аналогичных описанным ранее [6], нами зарегистрировано значительное превышение концентрации трития (табл. 1), что подтверждает течение процесса по реакции (2). Как показали контрольные измерения, образование трития не связано с проведением тлеющего разряда в среде дейтерия. В табл. 1 эксперименты № 2,4,5 проводились баз разряда, а концентрация трития была также высокая. После этого проведены эксперименты № 8-10 с порошком титана № 2 и с тлеющим разрядом на деформированной никелевой фольге. Для всех трех экспериментов зарегистрирована концентрация трития обычная для использованного дейтерия.

Во время этих экспериментов использовались порошки титана двух разных партий, № $1-$ не просеянный порошок, № $2-$ просеянная фракция $(80<d<150$ мкм).

Для определения дисперсного состава порошка титана был использован микроскопический анализ, который позволяет оценить не только геометрические размеры исследуемых объектов, но и увидеть особенности их формы, структуры и строения поверхности [7]. В результате микроскопического анализа были получены гистограммы распределения частиц по размерам. Для получения достоверных результатов определения размеров частиц был произведен замер 270 частиц в образце № 1 и 400 частиц в образце № 2.

Частицы порошка титана имеют форму, близкую к овальной, но также можно выделить частицы неправильной формы (рис. 1a,b). В обоих образцах присутствуют крупные и мелкие частицы. Результаты статистического анализа свидетельствуют о том, что исследуемые образцы порошка титана обладают достаточно широким диапазоном распределения частиц по размерам (рис. 2).

Видно, что в первом образце наибольшее количество частиц $(\approx 36 \%)$ имеет размер в пределах 75-100 мкм. 
Табл. 1. Концентрации трития в dpm (распады в минуту) для различных экспериментов.

Table 1. Concentration of tritium in dpm (decay in minute) for different experiments.

\begin{tabular}{|c|c|c|c|c|}
\hline № & $\begin{array}{l}\text { Материал } \\
\text { Material }\end{array}$ & $\begin{array}{l}\text { Порошок титана } \\
\text { Titanium powder }\end{array}$ & $c_{\mathrm{T}}, \mathrm{dpm}$ & $\begin{array}{l}\text { Режимы } \\
\text { Modes }\end{array}$ \\
\hline 1 & $\mathrm{Ni} \quad № 3$ & №1 & 12542.00 & 12.11 .2013 \\
\hline 2 & Ni №3 & №1 & 13102.00 & Без разряда / Without charge \\
\hline 3 & Ni №3 & №1 & 13674.00 & Заброс $\mathrm{T} 650^{\circ} \mathrm{C} /$ Excess of temperature to $650^{\circ} \mathrm{C}$ \\
\hline 4 & Ni №3 & №1 & 13638.00 & Без нагрева и разряда / Without heating and charge \\
\hline 5 & Ni №3 & №1 & 14240.00 & $T_{3} 400^{\circ} \mathrm{C}$ Без разряда / Without charge \\
\hline 6 & Ni №3 & №1 & 10939.00 & $T_{3} 400^{\circ} \mathrm{C}+$ разряд $/ \mathrm{T} 400^{\circ} \mathrm{C}+$ charge \\
\hline 7 & Ni №3 & №1 & 12159.00 & $T_{3} 500^{\circ} \mathrm{C}+$ разряд $/ T_{500^{\circ} \mathrm{C}+\text { charge }}$ \\
\hline 8 & Ni №7 & №2 & 1501.000 & $\mathrm{~T}_{3} 400^{\circ} \mathrm{C}+$ разряд $/ \mathrm{T} 400^{\circ} \mathrm{C}+$ charge \\
\hline 9 & $\begin{array}{l}\mathrm{Ni} \text { эл.хим.покр. } \\
\mathrm{Ni} \text { el.chem.coating }\end{array}$ & №2 & 1465.000 & $\mathrm{~T}_{3} 400^{\circ} \mathrm{C}+$ разряд $/ \mathrm{T} 400^{\circ} \mathrm{C}+$ charge \\
\hline 10 & $\mathrm{Ni} 6$ & №2 & 1571.000 & $T_{3} 400^{\circ} \mathrm{C}+$ разряд $/ \mathrm{T} 400^{\circ} \mathrm{C}+$ charge \\
\hline 11 & Ti №1 & №1 & 3900.00 & \\
\hline 12 & Ti №2 & №2 & 492.00 & \\
\hline
\end{tabular}

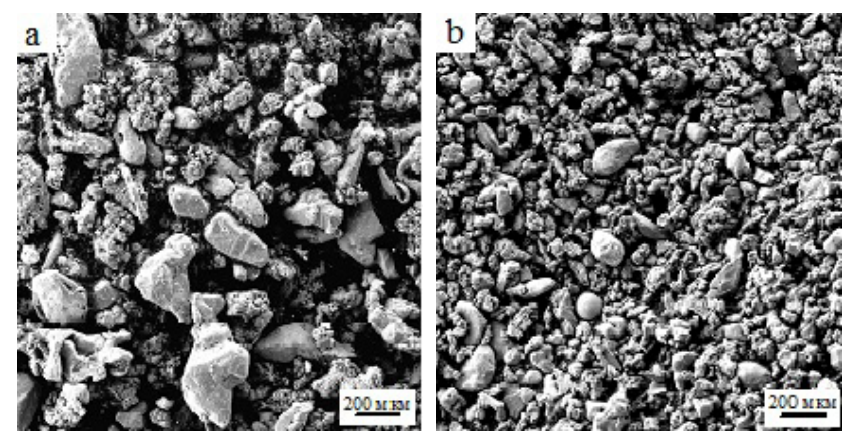

Рис. 1. Частицы порошка титана: а - образец № 1, b - образец № 2 .

Fig. 1. Particles of titanium powder: a - sample \# 1, b - sample \# 2 .

Доля крупных частиц размером 400 - 450 мкм составляет $\approx 0,6 \%$, доля мелких частиц размером 20 - 30 мкм составляет $\approx 8 \%$. Во втором образце наибольшее количество частиц $(\approx 23 \%)$ имеет размер около 75 мкм. Также наблюдаются крупные частицы, размер которых составляет 220 мкм $(\approx 0,5 \%)$ и 280 мкм ( $\approx 0,3 \%)$, мелкие частицы размером менее 30 мкм не наблюдаются. На основании полученных данных можно заключить, что средний размер частиц порошка титана в образце № 1 составляет $110 \pm 5$ мкм, в образце № $2-79 \pm 3$ мкм. Кроме того наблюдаются различия в структуре поверхности частиц. В обоих образцах встречаются частицы, имеющие как «плотную», так и «рыхлую» структуру. Частицы с «рыхлой» структурой представляют собой конгломераты более мелких частиц, размером $\approx 2$ мкм. «Рыхлая» структура, по-видимому, обладает значительной внутренней пористостью и дефектной кристаллической решеткой.

Микроскопический анализ проявляет наглядность размеров и формы частиц, но не позволяет учесть в распределении мелкие частицы. Дополнительная регистрация взвеси порошка титана в этаноле на лазерном дифракционном анализаторе размеров частиц позволила установить распределение мелких частиц (рис. 3).
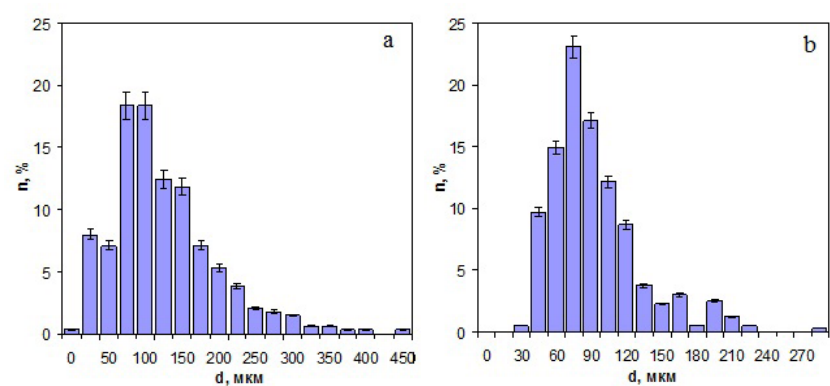

Рис. 2. Гистограмма распределения частиц порошка титана по размерам: $\mathrm{a}$ - образец № $1, \mathrm{~b}$ - образец № 2 ( $n$ - количество частиц порошка, \%; $d$ - размер частиц порошка, мкм).

Fig. 2. The histogram of size distribution of titanium powder particles: a - sample \# $1, \mathrm{~b}-$ sample \# 2 ( $n$ - amount of powder particles, \%; $d$ - size of powder particles, $\mathrm{mkm})$.

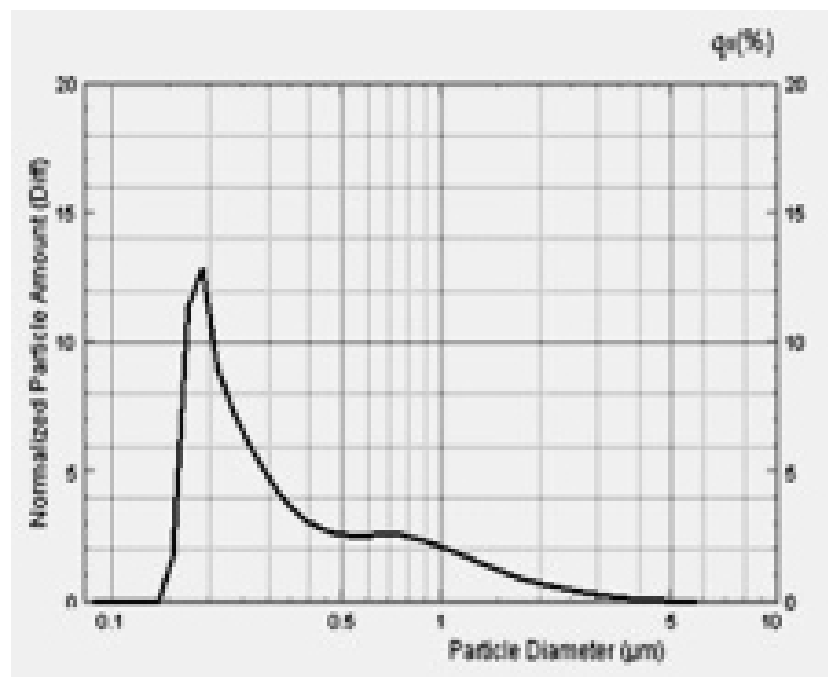

Рис. 3. Распределение размера частиц для суспензии порошка титана в этаноле.

Fig. 3. The size distribution of titanium powder particles in ethanol. 
Неизвестным параметром в предложенной модели [5] является поперечный размер длинномерных дефектов, возникающих в кристаллической решетке при поглощении дейтерия с взаимодействием дейтронов. По-видимому, существует оптимальный поперечный размер протяженных дефектов, при котором взаимодействие в них дейтронов протекает с высокой вероятностью. При меньшем размере дефектов будет происходить замедление движения дейтронов или их пар - квазиионов, при большем - уменьшится взаимодействие движущихся частиц с переменным кристаллическим полем, что приведет к уменьшению вероятности протекания переноса нейтрона в паре дейтронов.

Для порошка № 1, имеющего порошинки размером $d=0,2$ мкм, сфера, вписанная между плотно упакованными шарами, будет составлять порядка $d_{1}=3 \cdot 10^{-8} \mathrm{M}$ (0,3 мкм). Минимальный размер дефектов кристаллического строения - дислокаций близок к параметру кристаллической решетки, который для титана составляет $d_{2}=3 \cdot 10^{-9}$ м [8]. Таким образом, размер оптимальных дефектов для взаимодействия дейтронов в кристаллической решетке находится в диапазоне между этими величинами, но вероятно ближе к $d_{1}$.

Ранее проводились эксперименты по наработке трития на палладиевой мембране при тлеющем разряде в атмосфере дейтерия. Предварительно мембрану деформировали растяжением для увеличения плотности дислокаций с целью создать проницаемые для дейтронов дефекты. В ряде экспериментов зарегистрирована высокая температура мембраны [6], зафиксировано в этих экспериментах и повышенное содержание трития в дейтерии после окончания экспериментов $\left(c_{\mathrm{T}}, \mathrm{dpm}=1501 / \mathrm{мин}\right)$. Однако в экспериментах по насыщению спеченного порошка титана дейтерием получена концентрация трития в 80 раз большая $\left(c_{\mathrm{T}}, \mathrm{dpm}=12 \cdot 10^{3} 1 /\right.$ мин $)$, чем на палладиевой мембране.

Во время всех экспериментов уровни нейтронов $\left(N_{\text {пn }}=30 \pm 21 / 50\right.$ сек $)$ и гамма квантов
$\left(N_{\text {пу }}=700 \pm 151 / 5\right.$ сек $)$ не превысили обычные величины для данного помещения.

Можно предположить, что предполагаемые дефекты кристаллического строения в металлической матрице являются объектами NAE - Nuclear Active Environment, к поиску которых призывает E. Storms [3].

\section{4. Выводы}

Получены воспроизводимые результаты по образованию трития при поглощении дейтерия титановым порошком, подтверждающие протекание ядерной реакции.

Благодарность/Acknowledgements. Авторь признательны д.х.н. Мингалееву В.3. за измерение размеров частии, титана во взвеси порошка в этаноле на лазерном дифракиионном анализаторе.

\section{Литература/References}

1. Fleischmann M., Pons S. - J. Electroanal, 1989, v.261, pp. $301-308$.

2. Моррисон Д.Р. О. УФН 1991, т. 161, № 12, сс. 129 - 140

3. Storms E. The science of low energy nuclear reactions, New Jersey: World Scientific, 2007, 312 p.

4. Afonichev D.D., Murzinova M.A. Intern. J. Hydrogen Energy. 2003, v 28, No 9, pp 1005-1010.

5. Afonichev D. D. Intern. J. Hydrogen Energy. 2006, v 31, No 4, pp. $551-553$

6. Афоничев Д.Д., Галкин Е.Г., Хуснуллин А.М. Перспективные материалы, 2011, Сп. Выпуск (12), 06, cс. $37-41$.

7. Анциферов В.Н., Бобров Г.В., Дружинин Л.К. и др. Порошковая металлургия и напыленные покрытия: Учебник для вузов. М.: Металлургия, 1987. - 792 с.

8. Новиков И.И. Дефекты кристаллического строения металлов. Изд-во «Металлургия», 1975. 208 с. 